\title{
Peak cap stress calculations in coronary atherosclerotic plaques with an incomplete necrotic core geometry
}

\author{
Annette M. Kok ${ }^{1 *}$, Lambert Speelman ${ }^{1}$, Renu Virmani ${ }^{2}$, Antonius F. W. van der Steen ${ }^{1,3}$, Frank J. H. Gijsen ${ }^{1}$ \\ and Jolanda J. Wentzel ${ }^{1}$
}

*Correspondence:

a.m.kok@erasmusmc.nl

${ }^{1}$ Department of Cardiology,

Biomedical Engineering,

Erasmus MC, Rotterdam, The Netherlands

Full list of author information is available at the end of the article

\begin{abstract}
Background: Stress calculations in atherosclerotic coronary vulnerable plaques can aid in predicting coronary cap rupture. In vivo plaque geometry and composition of coronary arteries can merely be obtained via intravascular imaging. Only optical driven imaging techniques have sufficient resolution to visualize the fibrous cap, but due to limited penetration depth deeper components such as the backside of the necrotic core $(\mathrm{NC})$ are generally not visible. The goal of this study was to investigate whether peak cap stresses can be approximated by reconstructing the backside of the NC.

Methods: Manual segmentations of coronary histological cross-sections served as a geometrical ground truth and were obtained from seven patients resulting in 73 NCs. Next, the backside was removed and reconstructed according to an estimation of the relative necrotic core thickness ( $\mathrm{rNCt}$ ). The rNCt was estimated at three locations along the NC angle and based on either group averaged parameters or plaque specific parameters. Stress calculations were performed in both the ground truth geometry and the reconstructed geometries and compared.
\end{abstract}

Results: Good geometrical agreement was found between the ground truth NC and the reconstructed NCs, based on group averaged rNCt estimation and plaque specific rNCt estimation, measuring the NC area difference (25.1\% IQR 14.0-41.3\% and $17.9 \%$ IQR 9.81-32.7\%) and similarity index (0.85 IQR 0.77-0.90 and 0.88 IQR 0.79-0.91). The peak cap stresses obtained with both reconstruction methods showed a high correlation with respect to the ground truth, $r^{2}=0.91$ and $r^{2}=0.95$, respectively. For high stress plaques, the peak cap stress difference with respect to the ground truth significantly improved for the NC reconstruction based plaque specific features (6\%) compared to the reconstruction group averaged based (16\%).

Conclusions: In conclusion, good geometry and stress agreement was observed between the ground truth NC geometry and the reconstructed geometries. Although group averaged rNCt estimation seemed to be sufficient for the NC reconstruction and stress calculations, including plaque specific data further improved stress predictions, especially for higher stresses.

\section{Background}

The main cause of myocardial infarction is coronary plaque rupture. Not all coronary plaques will rupture; therefore it is of imminent importance to distinguish rupture prone

( ) 2016 Kok et al. This article is distributed under the terms of the Creative Commons Attribution 4.0 International License (http:// creativecommons.org/licenses/by/4.0/), which permits unrestricted use, distribution, and reproduction in any medium, provided you give appropriate credit to the original author(s) and the source, provide a link to the Creative Commons license, and indicate if changes were made. The Creative Commons Public Domain Dedication waiver (http://creativecommons.org/publicdomain/ zero/1.0/) applies to the data made available in this article, unless otherwise stated. 
plaques from stable plaques. Pathological studies showed that rupture prone plaques consists of a large necrotic core (NC), a thin fibrous cap and often are positively remodeled [21]. However, geometric and compositional information appeared to be insufficient to predict cardiovascular events, therefore new rupture risk parameters should be investigated [23].

From a biomechanical point of view, plaque rupture occurs when the stresses in the cap exceed the strength of the cap. Mechanical studies have shown that stress is an important indicator for plaque vulnerability $[10,24,26]$. Therefore, determining stresses in a cap can aid in predicting cap rupture. A common technique to calculate stresses is by using finite element analysis (FEA). In order to accurately calculate these stresses, blood pressure, material properties, and geometric factors are required. The most important geometric factor influencing peak cap stress (PCS) predictor is cap thickness, but also lumen size and shape and NC size and shape $[1,2,7,8,16,19]$.

To capture plaque geometry and composition in vivo, intravascular imaging is required. The only imaging techniques with sufficient resolution to accurately visualize the cap thickness are optical driven, such as optical coherence tomography. However, necrotic core tissue highly attenuates light, and thereby limits the penetration depth. Consequently, the NC geometry can become obscured, and thus is often only the front side of the NC visible. In order to allow FEA the complete geometry of the $\mathrm{NC}$ is essential.

The goal of this study was to investigate whether peak cap stresses can be approximated by reconstructing the backside of the NC. Therefore, we used a three-step approach: (1) a model was generated based on the histology data to estimate the NC thickness at several locations along the NC angle, (2) the backside of the NC was artificially removed and reconstructed based on characteristics of the NCs, (3) stresses and geometries of the plaque containing the reconstructed $\mathrm{NC}$ geometries were compared with the plaque containing the ground truth NC.

\section{Methods}

In order to reconstruct the backside of the NC, MATLAB (version 2014a, Mathworks Inc., Natick, MA, USA) was used. Six steps were taken: (1) manual segmentation of the coronary plaque geometry from histology ('ground truth'), (2) geometrical characterization was performed of all the plaques and NCs: to determine the group average (relative) $\mathrm{NC}$ thickness at the center (50\%) of the NC angle (midcap) and $\pm 25 \%$ of the NC angle (sidecaps) and corresponding plaque specific properties: cap thickness, NC angle, and intima-media thickness (IMT) were determined, (3) plaque specific relation between the relative $\mathrm{NC}$ thickness $(\mathrm{rNCt})$ and plaque specific properties was determined with a generalized estimation equation (GEE) model, (4) the backside of the NC geometry was removed and thereafter reconstructed based on group averaged rNCt estimation and a plaque specific rNCt estimation, (5) geometry assessment: the reconstructed NC geometries were compared with the ground truth geometry, and (6) the effect of the reconstruction on the PCS calculations was evaluated. All steps are explained in more detail below. 


\section{Histology}

Histology was used to obtain the NC geometry in atherosclerotic plaques to serve as the geometrical ground truth. It is important to obtain representative plaque cross-sections. Therefore, we used coronary plaque data of seven patients who died from severe coronary artery disease. The specimens were obtained from CVpath (CVPath Institute, Inc., Gaithersburg, MD, USA). Only those cross-sections that contained at least one NC were included. The coronary arteries were perfusion fixed in formalin at $100 \mathrm{mmHg}$ prior to the histological preparation. Each consecutive cross-section had a distance of at least $200 \mu \mathrm{m}$ to the next cross-section in the longitudinal direction. In total, 54 cross-sections (5 $\mu \mathrm{m}$ in thickness) were obtained from 13 different arteries of which 31 plaques included one necrotic core and 21 contained two NCs. When multiple NCs were present in a cross-section, the NCs were treated separately; this resulted in 73 plaque geometries. A Modified Movat pentachrome staining was used to identify different plaque components. The lumen, NC, intima, media and adventitia layer were manually delineated. The ground truth contours were finalized during a consensus meeting.

\section{Plaque and necrotic core characterization}

Geometrical characterization of the atherosclerotic plaque was performed by determining the geometrical properties of the plaque and the NC, see Fig. 1a. First, the minimum cap thickness and the front side of the $\mathrm{NC}$ were determined from the perspective of the lumen center, see Fig. 1b. Second, the NC angle was determined as the angle of the front side of the NC geometry, also with respect to the center of the lumen. Third, at three locations along the NC angle, the NC thickness was determined: at the center (50\%) of the NC angle (midcap) and $\pm 25 \%$ of the NC angle ( \pm sidecap). Also, the cap thickness (capT) and IMT were determined at these corresponding positions, see Fig. 1a.

\section{Group averaged necrotic core thickness estimation}

From the plaque and NC characterization we obtained group averaged NC thicknesses at three different locations: midcap and both sidecap positions. To make it robust for all plaque sizes, these NC thicknesses were divided by their corresponding IMT hereafter referred to as relative $\mathrm{NC}$ thickness $(\mathrm{rNCt})$. The median of the whole plaque group was calculated for the midcap position and the median was calculated for both sidecap

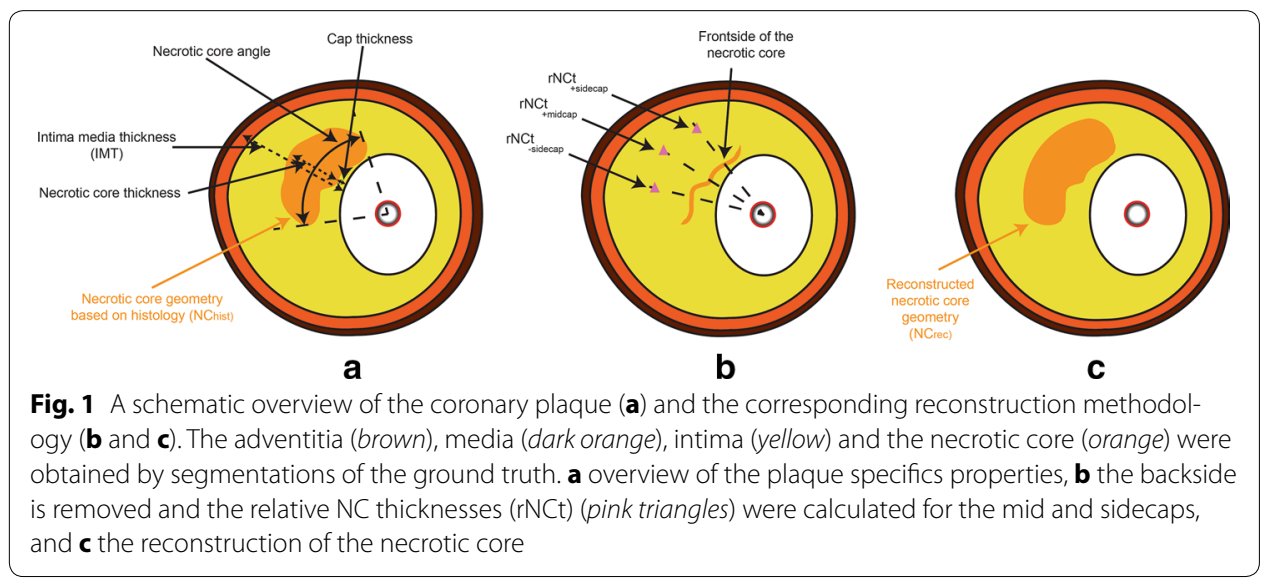


positions. These two $\mathrm{rNCt}$ were then used in the NC reconstruction, this method is hereafter referred to as the group averaged method.

\section{Plaque specific necrotic core thickness estimation}

Since, we assume that the NC thickness depends on plaque characteristics, we developed a method that estimates the NC thickness on a plaque specific level. Generalized estimation equation (GEE) model was used to determine a relationship between NC thickness and plaque specific parameters. In order to determine if each parameter in the model significantly contributed, the covariance matrix of $\beta$ was determined. This was done by a method previously described by Liang and Zeger et al. [15]. To also make the GEE model more robust, the rNCt was calculated. The plaque specific geometrical parameters used as input for the GEE model consist of NC angle, IMT, and cap thickness. We assume that the rNCt is symmetrical around the center of the NC angle, therefore two relationships were derived; one for the midcap and one for the combined sidecap positions. This relationship was defined as:

$$
\begin{aligned}
r N C t_{i}= & \beta_{0, i}+\beta_{N C \text { angle }, i} \cdot(N C \text { angle }[\mathrm{rad}])+\beta_{I M T, i} \cdot(I M T[\mu \mathrm{m}]) \\
& +\beta_{c a p T, i} \cdot(\operatorname{cap} T[\mu \mathrm{m}])
\end{aligned}
$$

with $\mathrm{i}$ indicating the relation at midcap or \pm sidecap and $\beta$ the constants determined by the GEE model. The GEE model was corrected for correlation amongst cross-sections within an artery [20]. Next, for each plaque the rNCt at the midcap and side cap positions was determined with the GEE model.

\section{Reconstruction}

Optical driven imaging techniques suffer from fatty tissues, since they highly scatter and absorb light, therefore limiting visualization beyond the NC. Thus, only the front side of the NC can be visualized reliably. To account for this only the front line of the NC was kept. This was done by drawing a line from the lumen center to each point in the NC. If the point was not the first intersection it was removed, in this way the backside of the $\mathrm{NC}$ was removed. The $\mathrm{NC}$ reconstruction was performed based on the group average rNCt estimations and the plaque specific rNCt estimation. The rNCt (at midcap and sidecaps) were converted to the absolute NC thickness and were placed at the corresponding positions. From histology we observed that NCs often have rounded features at the edges. Therefore, a part of a circle was attached to the edges of the NC, this also prevents geometric discontinuities in the reconstructed NC. The radius and angle of this part of the circle was empirically determined by optimizing the overlapping areas of all the reconstructed NCs with the ground truth areas. The optimized value for the angle was $30\left[^{\circ}\right]$ and the radius was dependent on the $\mathrm{NC}$ angle: $0.14 \times \mathrm{NC}$ angle [rad], these values were used for all the NC reconstructions. A visualization and description of how the center point of this circle was obtained is given in Fig. 2. Hereafter the backside is closed with the attached part of the circle by forcing the polynomial function to go through the side of the attached part of the circles (Fig. 1c). In case the polynomial function intersects the media layer $(\mathrm{n}=20$ for the group average $\mathrm{rNCt}, \mathrm{n}=8$ for the plaque specific rNCt estimation), the NC area outside the intima is removed. Since it is 


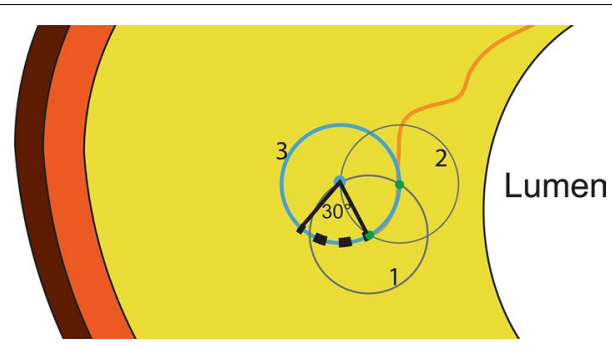

Fig. 2 Schematic illustration of the creation of the side of the NC using part of a circle. To calculate the center point of the circle used for creation of the side of the NC a combination of circles was used. The center of circle 1 was located at the edge of the necrotic core. One point in circle 1 intersects the cap, and this point is used as center point for circle 2. Then the two circles intersect at two locations, and the intersection most distant from the lumen was the center point of the final blue circle (3) for the side of the necrotic core (blue dot). Together with the empirically determined radius and angle the side can be reconstructed, see the black dotted line

not physiological that the $\mathrm{NC}$ is attached to the medial layer, a small layer was removed from the NC. In order not to alter the predicted geometry this layer was kept very small $(10 \mu \mathrm{m})$.

\section{Geometry analysis}

To investigate to what extent the reconstructed $\mathrm{NC}$ geometry corresponds with the histology based NC geometry, two measures were used: (1) the similarity index (SI) and (2) the relative difference in $\mathrm{NC}$ area, $\Delta \mathrm{A}(\%)$. The $\mathrm{SI}$ is a measure for overlap, defined as:

$$
S I=\frac{2 \cdot\left\{A_{G T} \cap A_{\text {rec }}\right\}}{A_{G T}+A_{\text {rec }}}
$$

with $\mathrm{A}_{\mathrm{GT}}$ the area of the ground truth $\mathrm{NC}$ geometry and $\mathrm{A}_{\mathrm{rec}}$ the area of the reconstructed NC geometry. To quantify mismatches in area the $\Delta \mathrm{A}(\%)$ :

$$
\Delta A(\%)=\frac{\left|A_{r e c}-A_{G T}\right|}{A_{G T}} \cdot 100 \%
$$

was used, with $\mathrm{A}_{\mathrm{GT}}$ the area of ground truth $\mathrm{NC}$ and $\mathrm{A}_{\text {rec }}$ the area of the reconstructed NC.

\section{Wall stress calculation}

Contours of the plaque with the ground truth NC and the reconstructed NC were imported and meshed in Abaqus for FEA (version 6.13, Dassault Systemes Simulia Corp., Providence, RI, USA). All material properties were assumed to be homogeneous and incompressible. Neo-Hookean material models for all components were used, as was done in similar parametric studies $[3,12,22]$. The mechanical properties of the components are listed in Table 1. To restrain rigid body motion, a compressible soft buffer around the model was used and the outer contour of the buffer was fully constrained. The material properties of the buffer are also listed in Table 1. Plaque stresses were computed by solving the mass and momentum equations. Linear tetrahedral elements were used with at least seven elements at the minimum thickness of the cap. One simulation of a $2 \mathrm{D}$ plaque took approximately $4 \mathrm{~h}$ with typically $1 \times 10^{6}$ elements on a 
Table 1 Material properties of the buffer and different components in the plaque

\begin{tabular}{lcllll}
\hline Material & E-modulus (kPa) & Poisson ratio (-) & $\mathbf{C}_{\mathbf{1 0}} \mathbf{( k P a )}$ & $\mathbf{D}_{\mathbf{1}}(\mathbf{-})$ & Reference \\
\hline Intima & 1000 & 0.498 & 166.7 & $1 \mathrm{e}-5$ & {$[11]$} \\
Media + adventitia & 1500 & 0.498 & 250 & $1 \mathrm{e}-5$ & {$[11]$} \\
Necrotic core & 6 & 0.498 & 1 & $1 \mathrm{e}-5$ & {$[17]$} \\
Buffer & 60 & 0.45 & 10 & 0.02 & {$[6]$} \\
\hline
\end{tabular}

$\mathrm{C}_{10}$ and $\mathrm{D}_{1}$ are constants to describe the Neo-Hookean model as used by Abaqus, representing the shear and bulk modulus as: $\mathrm{G}=2 \times \mathrm{C}_{10}$ and $\mathrm{K}=1 / \mathrm{D}_{1}$

standard desktop computer. Due to pressure fixation in the histology process, the histology based geometry was not stress free. Therefore, initial stresses up to $100 \mathrm{mmHg}$ were calculated with the backward incremental method [22]. Subsequently, a systolic blood pressure of $140 \mathrm{mmHg}$ was applied. PCS was defined as the maximum von Mises stress in the cap (defined as intima in front of the NC) and at the shoulders of the cap; defined as the area $15^{\circ}$ adjacent to the cap, see Fig. 3 [1]. Peak cap stresses were calculated and analyzed for the intima of the geometry containing the ground truth NC from histology $\left(\mathrm{PCS}_{\mathrm{GT}}\right)$, the reconstructed $\mathrm{NC}$ based on group average $\mathrm{rNCt}$ estimation $\left(\mathrm{PCS}_{\mathrm{GA}}\right)$ and the $\mathrm{NC}$ based on plaque specific $\mathrm{rNCt}$ estimation $\left(\mathrm{PCS}_{\mathrm{PS}}\right)$. The distance between the PCS location of the ground truth and the reconstructed plaque was determined. Hereafter co-localization was determined based on visual inspection. If the PCS was delocalized, displacement was classified as (1) shift from one side to the other side of the NC (2) slight delocalization or (3) translocation from the lumen to the NC side or vice versa.

\section{Statistics}

The data was tested for normality with a Kolmogorov-Smirnov test. If the data was normally distributed, mean and standard deviation are given and differences between data were tested with a paired t-test. Otherwise, the median and interquartile range were given and a paired non-parametric test (Wilcoxon signed ranktest) was used to test for significant differences. Statistical significance was considered as $\mathrm{p}<0.05$. The SI

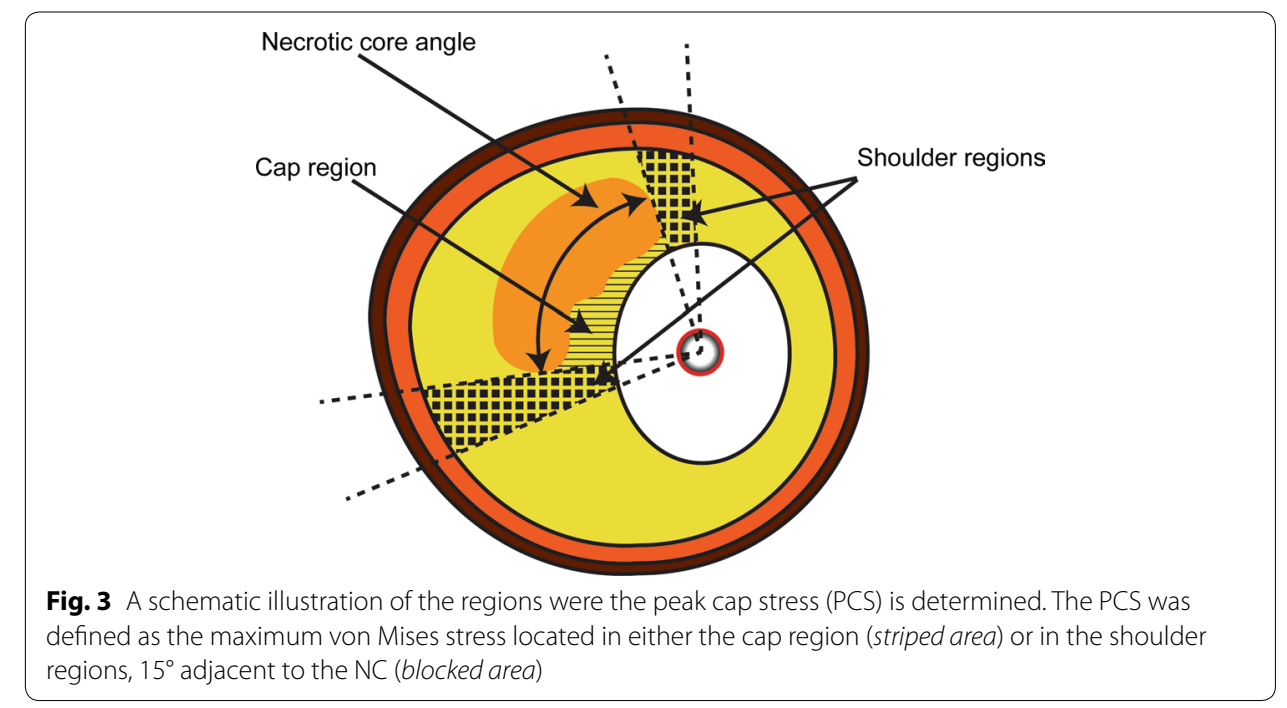


and $\triangle \mathrm{A} \%$ were analyzed for the geometry of the ground truth $\mathrm{NC}\left(\mathrm{NC}_{\mathrm{GT}}\right)$ vs. the reconstructed $\mathrm{NC}$ based on group averaged $\mathrm{rNCt}\left(\mathrm{NC}_{\mathrm{GA}}\right)$, and the reconstructed $\mathrm{NC}$ based on the plaque specific $\mathrm{rNCt}\left(\mathrm{NC}_{\mathrm{PS}}\right)$. Good similarity index was assumed to be at least $\mathrm{SI}>0.8$. Linear regression analysis was performed to compare peak wall stress of the different models.

\section{Results}

On average, the 73 plaques had a minimum cap thickness of $0.20 \mathrm{~mm}(0.09-0.40 \mathrm{~mm})$ and $\mathrm{NC}$ angle of and $54^{\circ}\left(35-75^{\circ}\right)$. At the midcap location, the cap thickness, IMT, and absolute NC thickness were $0.30 \mathrm{~mm}(0.13-0.53 \mathrm{~mm}), 1.05 \mathrm{~mm}(0.89-1.29 \mathrm{~mm})$, and $0.46 \mathrm{~mm}(0.27-0.54 \mathrm{~mm})$, respectively. For the sidecaps, the cap thickness, IMT, and absolute NC thickness were $0.31 \mathrm{~mm}(0.16-0.54 \mathrm{~mm}), 1.03 \mathrm{~mm}(0.84-1.24 \mathrm{~mm})$, and $0.36 \mathrm{~mm}(0.21-0.51 \mathrm{~mm})$. The median of the relative NC thicknesses at midcap and sidecap positions were 0.40 and 0.35 , respectively.

The GEE model showed a significant correlation between the estimated rNCt and the ground truth rNCt: at midcap $\left(\mathrm{r}^{2}=0.47\right)$ and at sidecap $\left(\mathrm{r}^{2}=0.44\right)$, see Table 2. All parameters (cap thickness, IMT and NC angle) were found to have a significant contribution for predicting the rNCt at both positions.

Figure $4 \mathrm{~A}-\mathrm{C}$ shows a typical example of the segmentation and reconstruction of the NC. The NC reconstruction based on group average (Fig. 4B) underestimated $(\mathrm{SI}=0.83)$ the $\mathrm{NC}$ area, whereas the plaque specific (Fig. $4 \mathrm{C}) \mathrm{NC}$ reconstruction predicted $(\mathrm{SI}=0.93)$ the $\mathrm{NC}$ area better. For all plaques, the $\mathrm{NC}_{\mathrm{GA}}$ matched the $\mathrm{NC}_{\mathrm{GT}}$ well with a SI and $\triangle \mathrm{A} \%$ of $0.85(0.77-0.90)$ and $25.1 \%(14.0-41.3 \%)$, respectively. The $\mathrm{NC}_{\mathrm{PS}}$ matched the $\mathrm{NC}_{\mathrm{GT}}$ even better with a SI and $\Delta \mathrm{A} \%$ of $0.88(0.79-0.91)$ and $17.9 \%$ (9.81$32.7 \%$ ), respectively. The $\mathrm{NC}$ geometries based on plaque specific $\mathrm{rNCt}$ had significantly better SI and $\Delta \mathrm{A} \%(\mathrm{p}<0.01)$ opposed to the $\mathrm{NC}$ geometry that were reconstructed solely based on group averaged rNCt.

For the typical example also the corresponding stress distribution in the fibrous cap of the ground truth geometry, and the reconstructed geometries are depicted in Fig. 4D-F. The $\mathrm{PCS}_{\mathrm{GA}}$ and $\mathrm{PCS}_{\mathrm{PS}}$ for this example showed 18 and $6 \%$ PCS difference with respect to the ground truth PCS. The absolute PCS values were $192 \mathrm{kPa}(82.1-355 \mathrm{kPa})$ for the ground truth, $192 \mathrm{kPa}(96.0-354 \mathrm{kPa})$ for the group average method and $196 \mathrm{kPa}(95.6-$ $369 \mathrm{kPa}$ ) for the plaque specific method. The overall difference in PCS between $\mathrm{PCS}_{\mathrm{GT}}$ vs. $\mathrm{PCS}_{\mathrm{GA}}$ was $15 \%(5-25 \%)$ and $\mathrm{PCS}_{\mathrm{GT}}$ versus $\mathrm{PCS}_{\mathrm{PS}}$ was $8 \%(5-23 \%)$. For all plaques, no significant difference was found in peak cap stresses between both reconstruction methods and the ground truth, only $\mathrm{PCS}_{\mathrm{GA}}$ versus $\mathrm{PCS}_{\mathrm{PS}}$ showed a significant difference $(\mathrm{p}<0.05)$. The correlation between the PCS of the ground truth and the PCS of the

Table 2 Generalized Estimation Equation (GEE) parameters with their standard errors

\begin{tabular}{|c|c|c|c|c|c|c|}
\hline & $\beta_{0}$ & $\beta_{\text {necrotic core angle }}$ & $\beta_{I M T}$ & $\beta_{c a p T}$ & $r^{2}$ & $p$ \\
\hline $\mathrm{rNCt}_{\text {midcap }}$ & $187 \pm 55.4$ & $63.3 \pm 26.6$ & $0.29 \pm 0.07$ & $-0.51 \pm 0.08$ & 0.47 & $<0.05$ \\
\hline $\mathrm{rNCt}_{ \pm \text {sidecap }}$ & $175 \pm 46.4$ & $130 \pm 19.8$ & $0.18 \pm 0.05$ & $-0.42 \pm 0.06$ & 0.44 & $<0.05$ \\
\hline
\end{tabular}

Predictive for the relative necrotic core thickness ( $r N C t)$, the $r^{2}$ and $p$ value of the regression line between the actual $r N C t$ value and the GEE model estimated value rNCt

All parameters with corresponding standard deviations are $\times 1000$-fold 


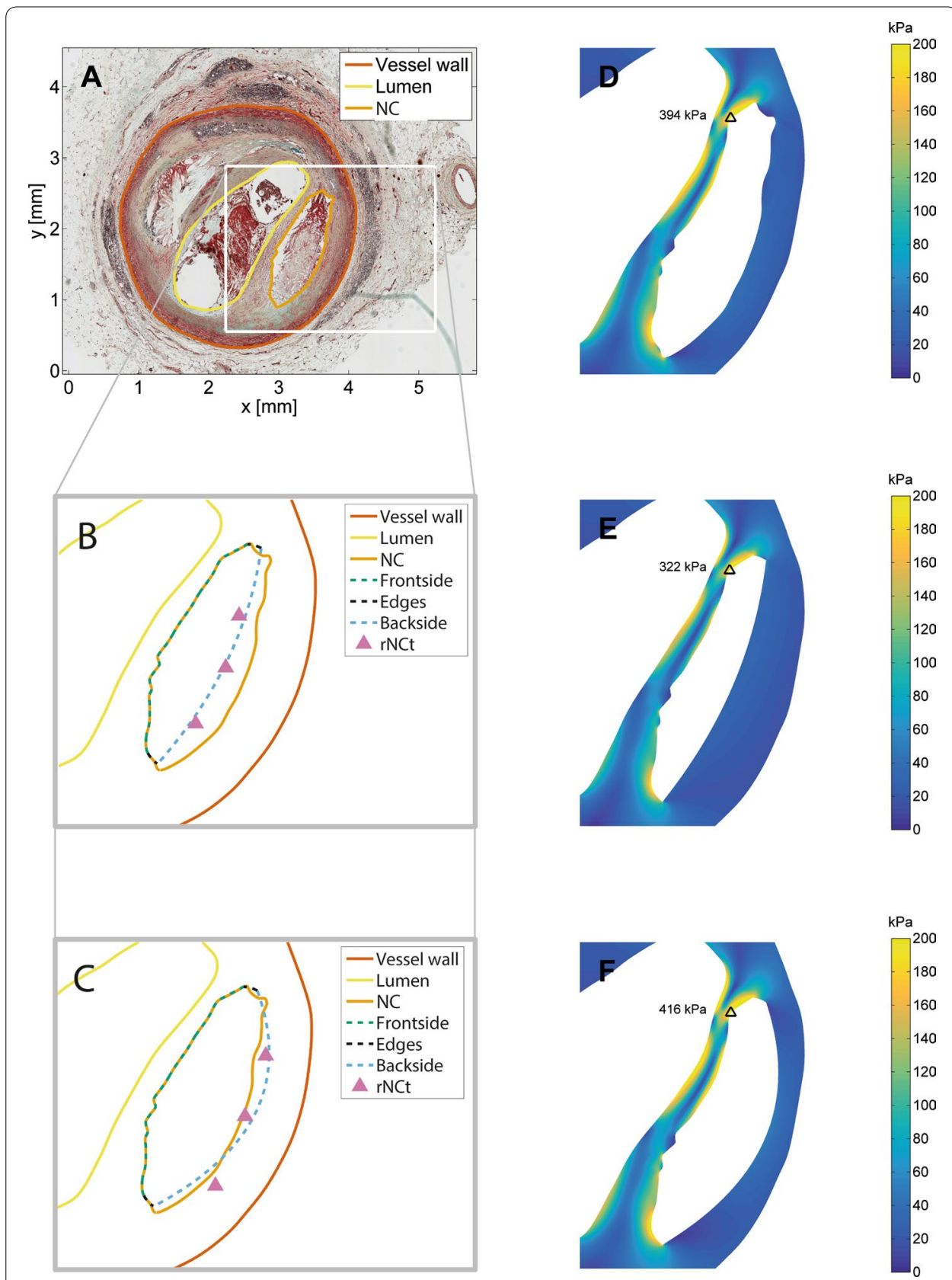

Fig. 4 From the histology image to stress calculations. A Cross-sectional slice from histology with the vessel wall, lumen, and necrotic core already delineated. B-F are zoomed versions of $(\mathbf{A})$ with necrotic core reconstruction based on the group averaged NC data $(\mathbf{B})$ and with necrotic core reconstruction based on the plaque specific (C) rNCt estimation methods. D-F are the wall stresses of the plaque containing the necrotic core geometry from the ground truth $(\mathbf{D})$, reconstruction of the necrotic core based on group averaged data $(\mathbf{E})$ and reconstruction of the necrotic core based on plaque specific $(\mathbf{F})$ method and the black triangles indicate the location of the peak cap stress

reconstructed geometries are shown in Fig. 5a, b. Both the $\mathrm{PCS}_{\mathrm{GA}}$ and $\mathrm{PCS}_{\mathrm{PS}}$ showed a

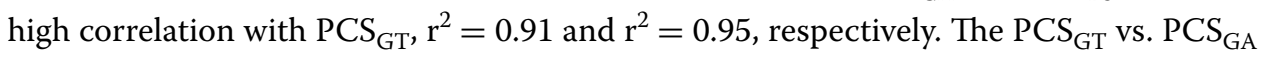
showed a higher offset and lower slope compared to the regression line obtained with the $\mathrm{PCS}_{\mathrm{GT}}$ vs. $\mathrm{PCS}_{\mathrm{PS}}$. 

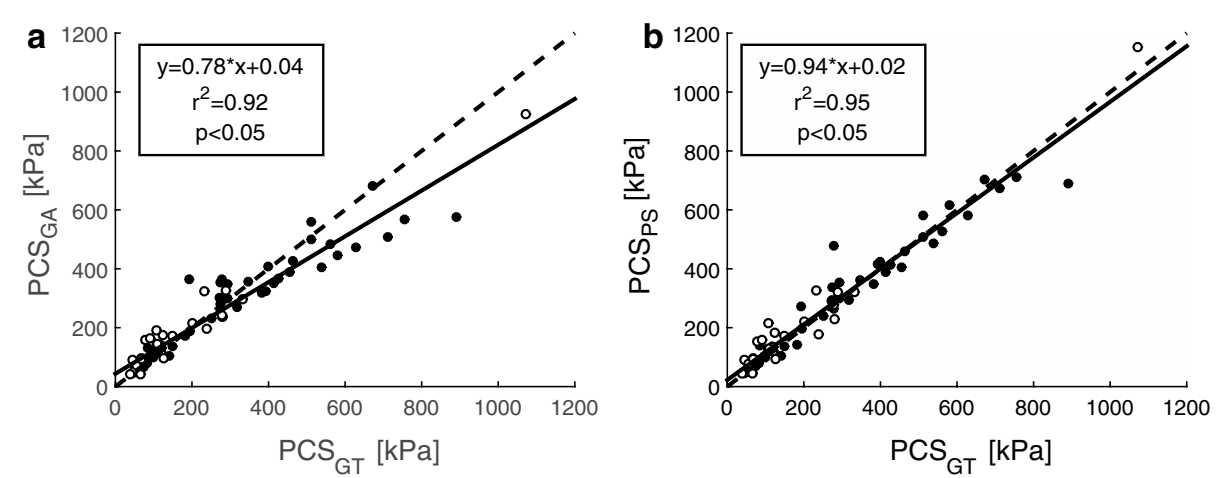

Fig. 5 The peak cap stress (PCS) of 73 plaque models from ground truth (histology) ( $x$-axis) versus the peak cap stress of the reconstructed necrotic core geometry (y-axis) are depicted. In a the PCS based on the NC reconstruction using group averaged rNCt estimation, $\mathrm{PCS}_{\mathrm{GA}}$ and $\mathbf{b} \mathrm{PCS}$ based on the NC reconstruction using the plaque specific rNCt estimation, $\mathrm{PCS}_{\mathrm{PS}}$. The closed circles indicate the cases for which the locations of the peak cap stresses was not shifted with respect to the ground truth, whereas the open circles did shift

In Fig. 5a, there seems to be a visual cut-off value at $300 \mathrm{kPa}$ were the group averaged method seems to underestimate the peak cap stresses. Coincidentally, this $300 \mathrm{kPa}$ threshold is also mentioned in literature as a rupture risk threshold [5]. In order to investigate if the high $\mathrm{PCS}_{\mathrm{GT}}$ were better estimated for the $\mathrm{PCS}_{\mathrm{PS}}$ than the $\mathrm{PCS}_{\mathrm{GA}}$, the crosssections were divided based on the PCS in a low $(<300 \mathrm{kPa})$ and high stress $(>300 \mathrm{kPa})$ group. Next, for both groups the PCS differences with respect to the ground truth were calculated. For the plaques with $\mathrm{PCS}_{\mathrm{GT}}$ below $300 \mathrm{kPa}$ both reconstruction methods showed $15 \%$ difference in PCS ( $\mathrm{p}>0.05$ ) compared to the ground truth. Interestingly, for the plaques with relative high $\mathrm{PCS}_{\mathrm{GT}}(>300 \mathrm{kPa})$, the PCS difference was $16 \%$ for the group averaged rNCt and only $6 \%$ for the plaque specific rNCt based reconstruction $(\mathrm{p}<0.05)$.

The distance between the locations of the PCS in the ground truth compared to the reconstructed geometries were evaluated. Since the distance between the location of the $\mathrm{PCS}_{\mathrm{GT}}$ and $\mathrm{PCS}_{\mathrm{GA}}$ was not different from the distance between the location of $\mathrm{PCS}_{\mathrm{GT}}$ and $\mathrm{PCS}_{\mathrm{PS}}(\mathrm{p}=0.29)$, only the distances between $\mathrm{PCS}_{\mathrm{GT}}$ and $\mathrm{PCS}_{\mathrm{PS}}$ are hereafter listed. In $67 \%(\mathrm{n}=49)$, the location of the $\mathrm{PCS}_{\mathrm{PS}}$ was similar as the location of the $\mathrm{PCS}_{\mathrm{GT}}$, with a distance of $4.13 \mu \mathrm{m}(2.66-17.6 \mu \mathrm{m})$, indicated by the closed circles in Fig. 5. For the remaining $33 \%$ of the data $(n=24)$, the location of the PCS was not similar and is depicted in Fig. 5 by the open circles. The shift in PCS was (1) from one side of the NC to the other side $(\mathrm{n}=9)$ with a distance of $0.99 \mathrm{~mm}(0.72-1.47 \mathrm{~mm})$ or $(2)$ at the same side $(\mathrm{n}=4)$ with a distance of $0.21 \mathrm{~mm}(0.07-0.66 \mathrm{~mm})$ or (3) translocated from the lumen side towards the front side of the NC or vice versa $(\mathrm{n}=11)$ with a distance of $0.92 \mathrm{~mm}$ $(0.24-1.32 \mathrm{~mm})$. For plaques that showed a shift in the peak stress location, the difference in PCS compared to the ground truth was larger (35\%) than for plaques with the same peak stress location $(11 \%)$. The $\mathrm{PCS}_{\mathrm{PS}}$ that were not at the same locations as the $\mathrm{PCS}_{\mathrm{GT}}$ had stresses mostly below $300 \mathrm{kPa}$ and represent the more stable plaque: $160 \mathrm{kPa}$ (93.9-226 kPa), respectively. For the $\mathrm{PCS}_{\mathrm{PS}}$ at the same location as the ground truth the stresses had a higher variation: $281 \mathrm{kPa}(102-392 \mathrm{kPa})$. 


\section{Discussion}

In this study, we analyzed the effect of the reconstruction of the missing NC backside with respect to geometry and stress calculations. The backside of the NC can be obscured due to limited penetration depth of in vivo light based imaging in coronary arteries. Histological cross-sections ('ground truth') were used to characterize the plaque and to reconstruct the $\mathrm{NC}$ geometry. Reconstruction was performed by determining the relative $\mathrm{NC}$ thickness $(\mathrm{rNCt})$ at three locations along the $\mathrm{NC}$ angle. The $\mathrm{rNCt}$ was either based on a group average or plaque specific parameters. Subsequently, the reconstructed $\mathrm{NC}$ geometries and the corresponding plaques from both methods were compared with the ground truth regarding the geometry and PCS. The following are the two main findings of this study: (1) high agreement was found between the NC geometries and the corresponding peak cap stresses of the reconstructed NCs and the ground truth, (2) reconstructing the $\mathrm{NC}$ based on average $\mathrm{NC}$ data resulted in underestimated peak cap stresses for high stress plaques only, whereas including plaque specific data for the NC reconstruction improved the stress prediction for these high stress plaques.

In terms of PCS prediction in the low PCS group $(<300 \mathrm{kPa})$, representing the more stable plaques, both $\mathrm{NC}$ reconstruction methods performed equally well, resulting both in $15 \%$ difference in PCS. This is relative low compared to changes in PCS in idealized models due to cap material properties (55-200 \%) [2]. In patient specific plaque geometries, the PCS showed to have two independent predictors: cap thickness and lumen curvature [1]. In the current study, the cap thickness and lumen geometry were unchanged, which may explain the relative good prediction of the peak cap stresses with the NC reconstruction methods.

A significant improvement in PCS was found for high PCS group ( $>300 \mathrm{kPa})$ using the plaque specific NC reconstruction method. This could be explained by the fact that an increase in NC area affects the PCS more in thin compared to thick fibrous caps [2]. Thus, it is likely that for high stress plaques, which have in general thin caps, a more precise reconstruction of the NC results would result in more accurate PCS prediction. Indeed, our data showed that for the plaques with a thin cap $(<0.20 \mathrm{~mm})$, (a) the corresponding $\mathrm{SI}$ and $\Delta \mathrm{A} \%$ significantly improved using plaque specific $\mathrm{NC}$ reconstruction method (SI: 0.83 vs 0.87 and $\triangle \mathrm{A} \% 25$ vs. $14 \%$ ) (b) the PCS estimation improved with respect to $\mathrm{PCS}_{\mathrm{GT}}$ when using plaque specific information (difference $\mathrm{PCS}_{\mathrm{GA}} 14 \%$ and $\mathrm{PCS}_{\mathrm{PS}} 7 \%$ ) whereas plaques with a thick cap did not show a significant improvement (15 vs. $18 \%)$.

For in vivo PCS computations in (vulnerable) coronary plaques, visualization of the cap is needed. The only possibility to obtain the cap thickness is with optical driven imaging techniques (e.g., optical coherence tomography, OCT), but it has limited penetration depth. Therefore the deeper lying tissues will become obscured, causing incomplete geometries for stress calculations. In this study, on purpose we investigated the influence of the reconstruction of the NC only. Using histology as ground truth enabled us to isolate the effects of reconstruction of the $\mathrm{NC}$ from possible other factors that can influence the PCS calculation. Logically, if the NC is obscured also the deeper laying vessel wall is missing. In a recent study it was shown that the vessel area in coronary arteries can be approximated by interpolating the non-obscured vessel wall [13]. Thus when 
moving forward to a clinical application the latter technique should be combined with the reconstruction of the NC to enable in vivo PCS calculations in coronary arteries.

Another attractive feature of OCT is that macrophages can be imaged. Extensive research showed that macrophages are linked with plaque instability $[14,18,25]$, therefore macrophages can be interpreted as a surrogate marker of cap strength. Co-localization of PCS and presence of macrophages might be instrumental in risk prediction. In that respect not only the PCS is of importance, but also the location. In this study a number of the PCS locations were not co-localized with the PCS $_{\mathrm{GT}}$ and thus might have consequences for risk prediction. However, most of the plaques with incorrect PCS locations showed low peak cap stresses, representing the more stable plaque.

Histological processing and variations in the manual segmentation might affect the ultimate geometry of the plaque and NC. We showed in an earlier study that histological processing hardly affects the geometry compared to the in vivo situation (Groen et al. 2010). However, since the model is tuned to this data-set, it is conceivable that variations because of the manual delineation and histological processing has some influence on the outcome of this model. We expect that the peak cap stress is minimally influenced, since we showed that the peak cap stress are hardly influenced by the exact geometry of the backside of the $\mathrm{NC}$, and we are therefore confident that the minor impact of intraand inter-observer variability on the model has also minor impact on the final stress. Therefore, it will not change the main conclusion of this study. Despite the relative large sample size of NCs $(n=73)$, the geometrical variation in the histology contours might not completely represent the whole population of NCs, due to the limited number of patients $(n=7)$. Little data is available on the geometrical variation of NC dimensions (e.g., lipid angle and $\mathrm{NC}$ thickness at midcap and sidecaps of the lipid angle). In a study by Gardner et al. [9], lipid cores were only assessed in plaques with a lipid core $>60^{\circ}$ in circumferential extent, $>200 \mu \mathrm{m}$ thick lipid core, and a mean fibrous cap thickness $<450 \mu \mathrm{m}$. They found a median minimum cap thickness of $164 \mu \mathrm{m}$ (IQR 101-243 $\mu \mathrm{m}$ ), a median lipid angle $102^{\circ}$ (IQR 77-132 $)$, and a median NC thickness of $448 \mu \mathrm{m}$ (IQR $315-565 \mu \mathrm{m})$ [9]. The NC thickness in our study showed a similar range $(461 \mu \mathrm{m}, \mathrm{IQR}$ 268-0.543 $\mu \mathrm{m})$, however, our median lipid angle was lower and showed less variation: $54^{\circ}$ (IQR $\left.35-75^{\circ}\right)$. This might be not so accurate to the estimation of the NC thickness and wall stress calculations for larger angles. However, no significant differences were found in PCS between plaques with a NC angle below or above the median NC angle of $60.9^{\circ}$ (20.6 and $\left.22.6 \%\right)$.

In the stress calculations of this study relative simple material models were chosen for the plaque components. In diseased tissue there are usually extra collagen fibers present to absorb higher stresses. Ideally, tissue nonlinearity and anisotropy should be taken into account. However, to the knowledge of the authors, experimental data representing anisotropy in coronary arteries is not available. Although in carotid arteries this data is available, the dispersion range is so high that modeling with isotropic model could suffice [4]. In addition to anisotropy and the geometric factors other factors will also influence the absolute values of PCS, such as, luminal pressure, initial stress, residual stress, $2 \mathrm{D}$ vs $3 \mathrm{D}$ assumptions, and material properties. The latter, might be the most important one, since it was shown that in an idealized geometry the PCS could change $200 \%$, just by changing the material properties of the intima. Since in this study in all models, the 
ground truth and reconstructed, the same material properties were used, no differences in absolute PCS based on the material properties may be expected. Therefore, conclusions on the reconstruction methodology and resulting differences in PCS still hold. However, when performing plaque specific risk analysis, nonlinearity and anisotropy will definitely impact the stresses.

In this study, we only retain the front line of the NC such that it resembles the optical driven imaging techniques. Another approach would be removing the NC behind a certain distance from the lumen. In this way, other features such as the edges of the NC and $\mathrm{NC}$ area change. It would be interesting to investigate to what extent these edges and area change affect the PCS calculation. However, it is arguable whether these methods will affect our results, since we already observed that a significant area difference did not result in a significant PCS change between the reconstruction methods. Another approach to refine our method in future work is to improve the GEE model by categorizing NC cores based on, size, shape, and location in the vessel wall. This refinement will probably improve the predictive value of the GEE model. However, we already showed that the PCS estimation between the group averaged and plaque specific method does not differ that much. Therefore, it is arguable whether this categorizing method will show an improvement in cap stress.

\section{Conclusions}

In conclusion, this study showed that reconstruction of the backside of a $\mathrm{NC}$ can be performed using either group averaged NC data or plaque specific NC data. A high correlation was found between peak cap stresses as obtained with the ground truth geometry and the peak cap stresses of both reconstruction methods. Although using group averaged NC data for the reconstruction of the backside of the NC performs well for the stress calculations, including plaque specific data in the $\mathrm{NC}$ reconstruction method improves the stress prediction, especially for high stress plaques.

\section{Authors' contributions}

AMK, LS, AFWS, FJHG and JJW have made substantial contributions to conception and design and analysis and interpretation of data. RV has made substantial contribution of the acquisition of data. AMK, LS, FJHG, RV, AFWS and JJW have been involved in drafting the manuscript or revising it critically for important intellectual content. All authors have given final approval of the version to be published. AMK and JJW agree to be accountable for all aspects of the work in ensuring that questions related to the accuracy or integrity of any part of the work are appropriately investigated and resolved. All authors read and approved the final manuscript.

Author details

${ }^{1}$ Department of Cardiology, Biomedical Engineering, Erasmus MC, Rotterdam, The Netherlands. ${ }^{2}$ CVPath, Gaithersburg, MD, USA. ${ }^{3}$ Department of Imaging Physics, Delft University of Technology, Delft, The Netherlands.

\section{Acknowledgements}

We thank A. C. Akyildiz, Ph.D. for the segmentation of the data. The research leading to these results has received funding from the European Research Council under the European Union's Seventh Framework Programme (FP/2007-2013)/ERC Grant Agreement n. 310457.

Competing interests

The authors declare that they have no competing interests.

Received: 29 October 2015 Accepted: 18 April 2016

Published online: 04 May 2016 


\section{References}

1. Akyildiz AC, Speelman L, Nieuwstadt HA, van Brummelen H, Virmani R, van der Lugt A, van der Steen AFW, Wentzel $J J$, Gijsen FJH. The effects of plaque morphology and material properties on peak cap stress in human coronary arteries. Comput Methods Biomech Biomed Engin. 2016;19:771-9.

2. Akyildiz AC, Speelman L, van Brummelen H, Gutiérrez MA, Virmani R, van der Lugt A, van der Steen AF, Wentzel JJ, Gijsen FJ. Effects of intima stiffness and plaque morphology on peak cap stress. Biomed Eng Online. 2011;10:25.

3. Badel P, Avril S, Sutton MA, Lessner SM. Numerical simulation of arterial dissection during balloon angioplasty of atherosclerotic coronary arteries. J Biomech. 2014;47:878-89.

4. Chai C-K, Speelman L, Oomens CWJ, Baaijens FPT. Compressive mechanical properties of atherosclerotic plaquesindentation test to characterise the local anisotropic behaviour. J Biomech. 2014;47:784-92.

5. Cheng GC, Loree HM, Kamm RD, Fishbein MC, Lee RT. Distribution of circumferential stress in ruptured and stable atherosclerotic lesions. A structural analysis with histopathological correlation. Circulation. 1993;87:1179-87.

6. Choi AP, Zheng YP. Estimation of Young's modulus and Poisson's ratio of soft tissue from indentation using two different-sized indentors : finite element analysis of the finite deformation effect. Med Biol Eng Comput. 2005:43:258-64

7. Finet G, Ohayon J, Rioufol G. Biomechanical interaction between cap thickness, lipid core composition and blood pressure in vulnerable coronary plaque: impact on stability or instability. Coron Artery Dis. 2004;15:13-20.

8. Gao H, Long Q. Effects of varied lipid core volume and fibrous cap thickness on stress distribution in carotid arterial plaques. J Biomech. 2008;41:3053-9.

9. Gardner CM, Tan H, Hull EL, Lisauskas JB, Sum ST, Meese TM, Jiang C, Madden SP, Caplan JD, Burke AP, Virmani R, Goldstein J, Muller JE. Detection of lipid core coronary plaques in autopsy specimens with a novel catheter-based near-infrared spectroscopy system. JACC Cardiovasc Imaging. 2008;1:638-48.

10. Gijsen FJH, Migliavacca F. Plaque mechanics. J Biomech. 2014;47:763-4.

11. Holzapfel GA, Sommer G, Gasser CT, Regitnig P. Determination of layer-specific mechanical properties of human coronary arteries with nonatherosclerotic intimal thickening and related constitutive modeling. Am J Physiol Heart Circ Physiol. 2005;289:H2048-58.

12. Kelly-Arnold A, Maldonado N, Laudier D, Aikawa E, Cardoso L, Weinbaum S. Revised microcalcification hypothesis for fibrous cap rupture in human coronary arteries. Proc Natl Acad Sci USA. 2013;110:10741-6.

13. Kubo T, Yamano T, Liu Y, Ino Y, Shiono Y, Orii M, Taruya A, Nishiguchi T, Shimokado A, Teraguchi I, Tanimoto T, Kitabata H, Yamaguchi T, Hirata K, Tanaka A, Akasaka T. Feasibility of optical coronary tomography in quantitative measurement of coronary arteries with lipid-rich plaque. Circ J. 2015;79:600-6.

14. Lendon CL, Davies MJ, Born GV, Richardson PD. Atherosclerotic plaque caps are locally weakened when macrophages density is increased. Atherosclerosis. 1991:87:87-90.

15. Liang K-Y, Zeger SL. Longitudinal data analysis using generalized linear models. Biometrika Trust. 1986;73:13-22.

16. Loree HM, Kamm RD, Stringfellow RG, Lee RT. Effects of fibrous cap thickness on peak circumferential stress in model atherosclerotic vessels. Circ Res. 1992;71:850-8.

17. Loree HM, Tobias BJ, Gibson LJ, Kamm RD, Small DM, Lee RT. Mechanical properties of model atherosclerotic lesion lipid pools. Arterios. 1994;14:230-4.

18. Moreno PR, Falk E, Palacios IF, Newell JB, Fuster V, Fallon JT. Macrophage infiltration in acute coronary syndromes, Implications for plaque rupture. Circulation. 1994;90:775-8.

19. Ohayon J, Finet G, Gharib AM, Herzka DA, Tracqui P, Heroux J, Rioufol G, Kotys MS, Elagha A, Pettigrew RI. Necrotic core thickness and positive arterial remodeling index: emergent biomechanical factors for evaluating the risk of plaque rupture. Am J Physiol Heart Circ Physiol. 2008;295:H717-27.

20. Ratcliffe SJ, Shults I. GEEQBOX : a MATLAB toolbox for generalized estimating equations and quasi-least squares. Stat Softw. 2008;25:1-14.

21. Schaar JA, Muller JE, Falk E, Virmani R, Fuster V, Serruys PW, Colombo A, Stefanadis C, Ward Casscells S, Moreno PR, Maseri A, van der Steen AFW. Terminology for high-risk and vulnerable coronary artery plaques. Report of a meeting on the vulnerable plaque, June 17 and 18, 2003, Santorini, Greece. Eur Heart J. 2004;25:1077-82.

22. Speelman L, Akyildiz AC, den Adel B, Wentzel JJ, van der Steen AFW, Virmani R, van der Weerd L, Jukema JW, Poelmann RE, van Brummelen EH, Gijsen FJH. Initial stress in biomechanical models of atherosclerotic plaques. J Biomech. 2011:44:2376-82.

23. Stone GW, Maehara A, Lansky AJ, de Bruyne B, Cristea E, Mintz GS, Mehran R, McPherson J, Farhat N, Marso SP, Parise $H$, Templin B, White R, Zhang Z, Serruys PW. A prospective natural-history study of coronary atherosclerosis. N Engl J Med. 2011;364:226-35.

24. Tang D, Teng Z, Canton G, Hatsukami TS, Dong L, Huang X, Yuan C. Local critical stress correlates better than global maximum stress with plaque morphological features linked to atherosclerotic plaque vulnerability: an in vivo multipatient study. Biomed Eng Online. 2009;8:15.

25. Van der Wal AC, Becker AE, van der Loos CM, Das PK. Site of intimal rupture or erosion of thrombosed coronary atherosclerotic plaques is characterized by an inflammatory process irrespective of the dominant plaque morphology. Circulation. 1994:89:36-44

26. Wang L, Zheng J, Maehara A, Yang C, Billiar KL, Wu Z, Bach R, Muccigrosso D, Mintz GS, Tang D. Morphological and stress vulnerability indices for human coronary plaques and their correlations with cap thickness and lipid percent: an IVUS-based fluid-structure interaction multi-patient study. PLoS Comput Biol. 2015;11:1-15. 